\title{
INDICADORES DE DESEMPENHO PARA O SISTEMA DE PRODUÇÃO ENXUTO
}

\section{PERFORMANCE MEASURES FOR LEAN PRODUCTION SYSTEM}

\author{
Edwin Cardoza \\ Universidade de São Paulo - Escola de Engenharia de São Carlos - Programa de Pós-graduação do \\ Departamento de Engenharia de Produção. Av. Trabalhador São-carlense n.400, São Carlos - SP. \\ Tel. (16) 3373-8287 - e-mail: edwin@sc.usp.br
}

\section{Luiz C. Ribeiro Carpinetti}

Universidade de São Paulo - Escola de Engenharia de São Carlos - Departamento de Engenharia de Produção. Av. Trabalhador São-carlense n.400, São Carlos - SP.

Tel. (16) 3373-9421 - e-mail: carpinet@ prod.eesc.usp.br

\section{RESUMO}

No início do século XXI, é notório que uma série de paradigmas industriais está sendo revista e alterada pelas empresas e universidades. Entre as mudanças mais importantes destaca-se o processo de transição da produção em massa para a produção enxuta. A literatura específica da área de gestão de operações demonstra que existem diferenças significativas nesses métodos de produção. Uma das diferenças é o tipo de indicador de desempenho (ID) que é utilizado pelos gerentes de manufatura para avaliar os processos operacionais das fábricas. Porém, nota-se que existem poucas informações sobre como desenvolver os ID's no sistema de produção enxuto. Considerando essa questão, o objetivo deste estudo é discutir o processo de desenvolvimento e implantação dos ID's nesse sistema de produção. Apresentar-se-á de forma conclusiva um conjunto de indicadores de desempenho utilizados por empresas do setor agrícola que implantaram os conceitos desse novo sistema de manufatura e algumas considerações sobre os ID's utilizados na produção enxuta.

Palavras-chave: Produção Enxuta; Indicadores de Desempenho.

\begin{abstract}
In the beginning of the XXI century, it has noticed that the paradigms for operation are review and altered by companies and universities. Among relevant changes, it is important to highlight the transition process of mass production to lean production system. A survey in the literature into operations management demonstrated that the differences between production practices exist mass production and lean production. One difference is performance measures (PM) that will be used by several managers to manage industrial operational process. However, it has noticed that few information exist on how to develop the performance measures to lean manufacturing system. Considering this question, the objective of this paper is to discuss a development process and implantation of the performance measures for this production system. The illustration of a group of performance measurement used by companies that implanted the concepts of this new model of manufacturing is presented and some considerations on performance measurement and lean production.
\end{abstract}

Keywords: Lean Production; Performance Measures. 


\section{INTRODUÇÃO}

As exigências dos consumidores e o crescimento dos concorrentes (competitividade dinâmica) obrigam as empresas a buscar novas práticas de manufatura. No início da década de 1960, essa dinâmica levou as empresas japonesas do setor automobilístico, em especial a Toyota Motor Company, a desenvolver métodos diferentes de fabricar veículos em relação aos utilizados pela indústria americana, na época onde o destaque era o sistema de produção em massa da Ford Company e General Motor (OHNO, 1997; WOMACK et al., 1992; CUSUMANO, 1989). A quebra desse paradigma resultou em um novo modelo de sistema de produção, conhecido como Sistema de Produção Enxuto ou Sistema Toyota de Produção (Lean Manufacturing/Lean Production).

Segundo Ohno (1997), o foco da produção enxuta é a absoluta eliminação ou redução do desperdício. Isso envolve mudanças nas práticas de gestão de qualidade e gestão de operações utilizadas para melhorar e gerenciar os processos produtivos. Uma das alterações mais significativas diz respeito ao conjunto de indicadores de desempenho (ID's) que são utilizados pelos gerentes de manufatura para avaliar a qualidade industrial do processo e dos produtos, o inventário dos estoques em processos (work-in-process - WIP), a produtividade da célula, o tempo de fabricação, o tempo de preparação (setup), a satisfação dos clientes e dos funcionários, entre outros.

Entretanto, na literatura específica da área de gestão de operações e melhoria da qualidade existem poucas informações sobre como desenvolver tais ID's nesse sistema de manufatura. Considerando esse aspecto, o objetivo deste estudo é discutir o processo de desenvolvimento e a implantação dos indicadores de desempenho do Sistema de Produção Enxuto.

Nos itens que seguem serão apresentados os conceitos de produção enxuta, avaliação de desempenho e a descrição do processo de desenvolvimento dos indicadores. Apresentar-se- 
á uma lista de indicadores utilizados por empresas que implantaram os conceitos de produção enxuta no ano de 2003. Para finalizar, são realizadas algumas considerações sobre o processo de gestão de avaliação de desempenho e produção enxuta.

\section{SISTEMA DE PRODUÇÃO ENXUTO}

A base de funcionamento do Sistema de Produção Enxuto é formada pelo método do tempo propício lust-in-time - JIT) e da autonomação (Jidoka). O JIT determina que as empresas necessitam eliminar ou reduzir os estoques de produtos e devem procurar trabalhar em parceria com seus fornecedores a_fim de nivelar e evitar o excesso de produção. O Jidoka é o conjunto de práticas que fornecem aos equipamentos e, principalmente, aos operadores da produção a habilidade de detectar quando uma condição anormal ocorre e interrompe imediatamente o trabalho (OHNO, 1997).

Womack, et al. (1992) e Ohno (1997) ressaltam que esse sistema inovador é uma resposta das empresas japonesas ao sistema de produção em massa utilizado pela indústria americana. As mudanças surgiram porque esse processo demanda altos investimentos nos equipamentos e a dedicação exclusiva dos mesmos às linhas de produção, utiliza profissionais excessivamente especializados, elevados investimento nos moldes de fabricação e é um processo limitado para atender as necessidades dos clientes. O resultado é a baixa flexibilidade das linhas de produção em atender as mudanças solicitadas pelos clientes com relação aos produtos.

É importante ressaltar que essas limitações apareceram pelas próprias características do mercado doméstico japonês (poucos recursos, problemas de qualidade etc.) e não significam que a produção em massa seja um modelo em desuso ou ultrapassado sem vantagens industriais, conforme apresentado por Cooney (2002).

No Sistema de Produção Enxuto um dos objetivos é receber e produzir pequenos lotes de produtos. Neste caso, o raciocínio é que uma estação de trabalho só produz para a 
próxima estação quando o solicita ou avisa que o componente foi utilizado. Na maioria das vezes o fluxo da informação ocorre por meio de um dispositivo visual que autoriza e dá instruções para a produção, conhecido como cartões Kanban.

Segundo Cusumano (1989), para conseguir fabricar lotes menores de produção é necessário reduzir o tempo de ressuprimento (lead time), o tempo de preparação (setup), criar uma produção balanceada, modificar a rotina de trabalho das pessoas, eliminar os desperdícios e o tempo de movimentação dos funcionários. Os sete desperdícios mais comuns nas empresas são: superprodução, esperas, defeitos de qualidade, inventários desnecessários, processamento inapropriado, transporte excessivo e movimentação desnecessária OHNO, 1997).

Warnecke e Hüser (1995) definem, também, que a produção enxuta é representada por um sistema de avaliação de desempenho e pelas práticas de melhoria contínua. Segundo os autores, quando os métodos de melhoria são estendidos para todos os setores da empresa (desenvolvimento de produtos, cadeia de suprimentos, processos de manufatura e serviços oferecidos antes e depois das vendas), os desperdícios são eliminados e se pode alcançar um desempenho melhor nos processos produtivos e de negócio. Além disso, nas empresas existe a tendência de expandir esses conceitos aos seus fornecedores, formando assim estratégias competitivas que criam perspectivas de crescimento e domínio do mercado.

Segundo Standard e Davis (1999), todas essas características determinam que as empresas e, principalmente, as novas linhas de produção devem ser gerenciadas com novos indicadores de desempenho integrados com os indicadores financeiros.

\section{AVALIAÇÃO DO DESEMPENHO}

A partir do momento que as mudanças tecnológicas e as forças do mercado (demanda e competitividade) passaram a pressionar as empresas, percebeu-se que os modelos de avaliação de desempenho tradicionais (baseado unicamente em indicadores financeiros) 
eram limitados para gerenciar os negócios. Segundo Neely (1998), no atual cenário empresarial os novos modelos de avaliação de desempenho são necessários para: verificar e comunicar a posição no mercado; motivar o progresso e o comprometimento dos funcionários com as mudanças ou projetos de melhoria que são implantados; e para auxiliar na tomada de decisão sobre o processo de implantação e gerenciamento das melhorias e mudanças empresariais.

Os modelos descritos na literatura específica da área de avaliação de desempenho e os mais utilizados pelas empresas para desenvolver tais indicadores são: Balanced Scorecard (KAPLAN e NORTON, 1996), Performance Prism (NEELY e ADAMS, 2001), Integrated Performance Measurement Systems (BITITCI et al., 1997) e Performance Pyramid (CROSS e LYNCH, 1990). Todos esses modelos apresentam os objetivos, as áreas críticas de desempenho, os processos e os tipos de indicadores que devem ser utilizados pelos gerentes para avaliar o desempenho organizacional.

Paralelamente, surgiram os procedimentos de implantação dos projetos de avaliação do desempenho (RENTES et al., 2002; MARTINS, 2002; VAN AKEN et al., 2001; NEELY et al., 2000; BITITCI et al., 2001; FLAPPER et al., 1996). A figura 1 apresenta as etapas envolvidas no desenvolvimento (projeto) e na utilização (implantação) do sistema de avaliação de desempenho - SMD (CARDOZA et al., 2003).

A proposta desses autores é apresentar uma sistemática para integrar os objetivos das unidades de negócios ou dos processos administrativos com os indicadores de desempenho utilizado nos processos operacionais. Além disso, é importante ressaltar que essa proposta surgiu porque se verificou que na literatura da área de medição de desempenho, apesar do número elevado de referências bibliográficas, são poucos os trabalhos que abordam simultaneamente as duas fases do projeto de desenvolvimento de indicadores. 


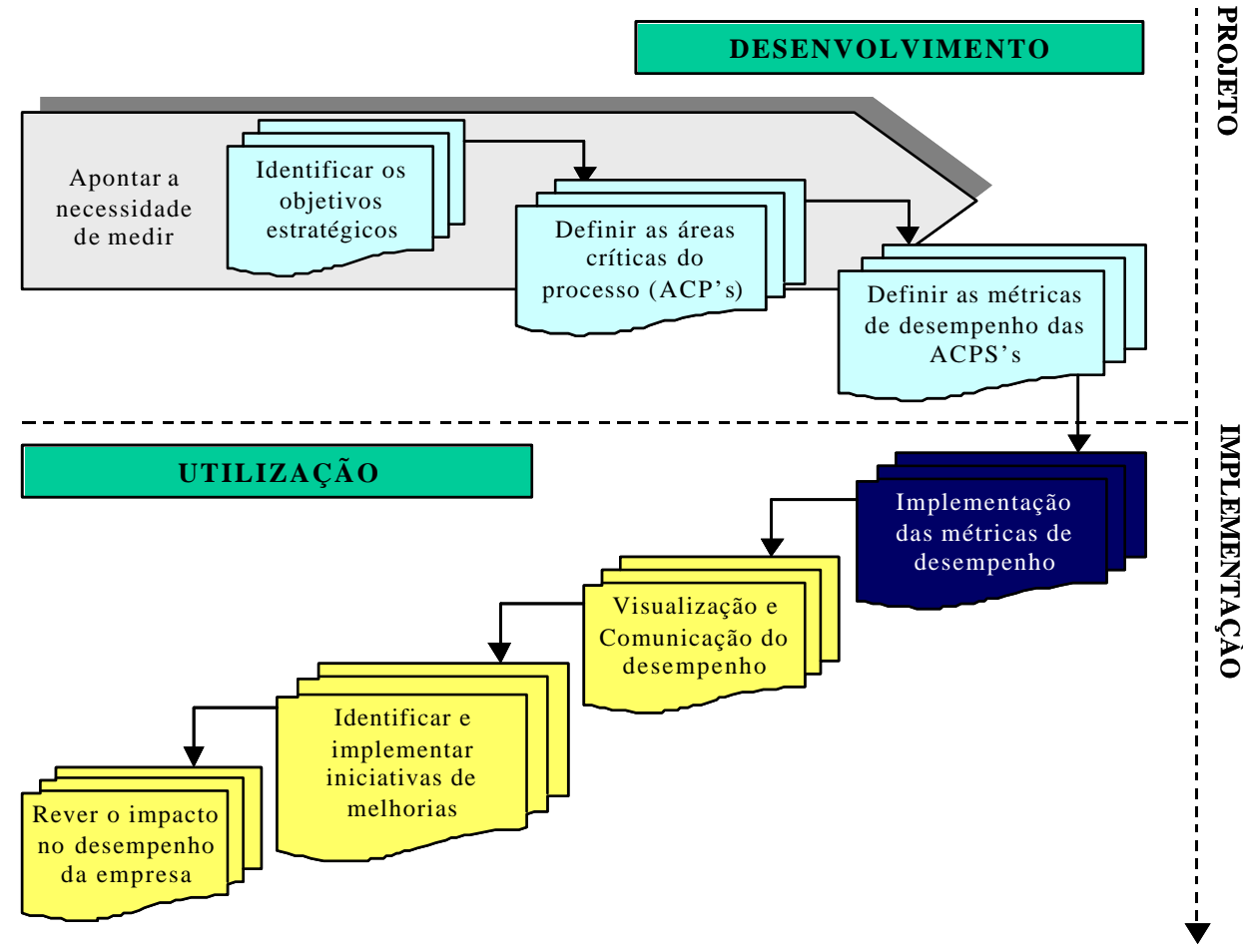

Figura 1: Etapas de desenvolvimento, implantação e utilização dos SMD’s

Fonte: Cardoza, et al. (2003).

Outro aspecto discutido nessas pesquisas é a Gestão Visual do sistema de avaliação de desempenho. Ou seja, como é importante que as informações (qualidade, controle de produção, atrasos na entrega, demanda dos componentes, produtividade etc.) estejam visíveis para os usuários do sistema de informação.

Considerando as tendências e os desafios que as empresas estão enfrentando no início do século XXI: demanda variável, baixos estoques, diminuição do tempo de ressuprimento global de produção, ciclos de vida dos produtos menores, produtos customizados etc., nota-se que cada vez mais será necessário utilizar métodos para desenvolver indicadores de desempenho flexíveis e dinâmicos que se adaptem as mudanças dos sistemas de manufatura.

\section{AVALIAÇÃO DO DESEMPENHO NA PRODUÇÃO ENXUTA}

Segundo Ohno (1997), um dos maiores problemas das fábricas é que os setores criam visões diferentes sobre a companhia como um todo. Os problemas que surge m com esse tipo 
de configuração é que dificulta a tomada de decisão e a identificação das restrições que cada setor possui, dificilmente é definido como a informação (métricas de desempenho) será utilizada (MARTINS, 2002) e faltam métodos para assegurar a coleta, análise, interpretação e disseminação dos dados na empresa (NEELY et al., 2000), existe uma integração frágil entre os objetivos estratégicos e processo críticos de negócios (BITITCI et al., 2001) e as informações não retratam os resultados das ações de melhoria (KAPLAN e NORTON, 1996).

Uma vantagem alcançada quando desenvolvido e implantado os conceitos de avaliação de desempenho no sistema de produção enxuto é a quantidade de informações que são reunidas das diversas áreas da empresa. Com isso é possível identificar com mais segurança os problemas da fábrica e elaborar os eventos de melhoria (kaizen) com mais eficiência.

Os ID's são implantados simultaneamente com algumas das metodologias propostas pela produção enxuta: layout da manufatura, gestão da demanda, planejamento da produção, logística integrada (abastecimento interno e externo), milk run (um método de acelerar o fluxo de materiais entre as unidades industriais no qual os veículos seguem uma rota para fazer múltiplas cargas e entregas em muitas unidades industriais), Gestão de Conhecimento, Sistema de Movimentação etc. (ROTHER e SHOOK, 1999).

Pode-se afirmar que o sistema de produção enxuto é um conjunto de projetos ou subprojetos que são implantados e integrados com o objetivo de aumentar o desempenho organizacional e, principalmente, alterar (mudar) os indicadores financeiros da empresa. Algumas vezes esse princípio não ocorre automaticamente, conforme comprovado por Lewis (2000). Entretanto, o autor ressalta que essa questão depende do tipo de empresa e, principalmente, do tipo de produto. Além disso, a metodologia utilizada para o desenvolvimento e a implantação dos conceitos é um aspecto que também influenciam o sucesso do projeto enxuto. 
Assim, é evidente que o tema de produção enxuta e avaliação de desempenho estão relacionados e ambos influenciam os processos administrativos e operacionais das empresas. Destaca-se que essa integração permite com que o sistema de manufatura consiga absorver e reagir rapidamente às transformações do mercado (demanda variável).

\subsection{Tipos de indicadores de desempenho}

O quadro 1 apresenta a compilação de alguns ID's que podem ser utilizados no Sistema de Produção Enxuto (SANCHEZ e PEREZ, 2001; STANDARD e DAVIS, 1999). A seta representa a alteração ou a mudança da métrica (do desempenho) quando aplicados os conceitos de produção enxuta.

Quadro 1: Indicadores de desempenho propostos para o sistema de produção enxuto

\begin{tabular}{|l|c|}
\hline \multicolumn{1}{|c|}{ Definição } & Alteração \\
\hline percentual de peças comuns para os produtos da empresa & $?$ \\
\hline giro do estoque & $?$ \\
\hline número de vezes que as peças são movimentadas & $?$ \\
\hline distância que as peças percorrem na fábrica & $?$ \\
\hline percentual da manutenção preventiva versus a manutenção total & $?$ \\
\hline número de sugestões dos empregados por ano & $?$ \\
\hline valor do refugo e re-trabalho com relação as vendas da empresa & $?$ \\
\hline porcentagem de tempo do equipamento parado por mau funcionamento & $?$ \\
\hline porcentagem de sugestões implantadas & $?$ \\
\hline ganhos ou benefícios alcançados com as sugestões implantadas & $?$ \\
\hline tempo de ressuprimento (lead time) do pedido dos clientes & $?$ \\
\hline número de fornecedores para os componentes mais importantes & $?$ \\
\hline porcentagem de empregados trabalhando em equipes & $?$ \\
\hline porcentagem e número de tarefas realizadas pelas equipes & $?$ \\
\hline tamanho do lote de produção & $?$ \\
\hline inspeções visuais no controle de qualidade & $?$ \\
\hline controle estatístico do processo & $?$ \\
\hline controle kanban da produção & $?$ \\
\hline número de testes de qualidade das peças & $?$ \\
\hline tempo de preparação (setup) do processo & $?$ \\
\hline porcentagem das peças recebidas dos fornecedores pelo sistema JIT & $?$ \\
\hline número de sugestões de melhoria realizadas pelos fornecedores & $?$ \\
\hline
\end{tabular}

Para Sanchez e Perez (2001) são seis os grupos de indicadores que podem ser classificados e implantados de acordo com as práticas do projeto enxuto: formação de equipes 
multifuncionais, eliminação de atividades que não agregam valor, melhoria contínua, JIT de

produção e de entrega, integração de fornecedores e de vantagem competitiva. Ainda apontam

que uma lista de verificação (checklist) deve ser realizada para examinar se realmente essas

práticas estão sendo avaliadas por indicadores de desempenho.

O quadro 2 apresenta os principais indicadores utilizados por um conjunto de empresas (fábricas de máquinas agrícolas) que implantaram os conceitos de produção enxuta no ano de 2003, todas estão localizadas no interior do Estado de São Paulo.

Quadro 2: ID's do sistema de produção enxuto de fábricas de máquinas agrícolas

\begin{tabular}{|c|c|c|}
\hline Indicador & Definição & Fórmula \\
\hline $\begin{array}{l}\text { Índice de entrega ao } \\
\text { cliente-fornecedor }\end{array}$ & $\begin{array}{l}\text { o objetivo é acompanhar a capacidade da empresa (atraso) } \\
\text { de entregar peças ao cliente-fornecedor (operação } \\
\text { terceirizada) }\end{array}$ & $\begin{array}{l}\text { número de pedidos entregues / } \\
\text { número de pedidos solicitados }\end{array}$ \\
\hline $\begin{array}{l}\text { Índice de atrasos do } \\
\text { cliente-fornecedor }\end{array}$ & $\begin{array}{l}\text { o objetivo é acompanhar o cliente-fornecedor que atrasa o } \\
\text { abastecimento de componentes }\end{array}$ & $\begin{array}{l}\text { número de dias que o fornecedor } \\
\text { está em atraso }\end{array}$ \\
\hline $\begin{array}{l}\text { Itens comerciais } \\
\text { faltando na linha de } \\
\text { produção }\end{array}$ & $\begin{array}{l}\text { o objetivo é medir a eficiência do sistema de controle da } \\
\text { produção }\end{array}$ & $\begin{array}{l}\text { número de itens comerciais em } \\
\text { atraso na linha de produção }\end{array}$ \\
\hline $\begin{array}{l}\text { Causas das paradas } \\
\text { da linha de } \\
\text { produção }\end{array}$ & $\begin{array}{l}\text { o objetivo é identificar quais são os itens que faltaram na } \\
\text { linha de produção }\end{array}$ & $\begin{array}{l}\text { para cada parada é descrita a } \\
\text { causa da interrupção da produção }\end{array}$ \\
\hline $\begin{array}{l}\text { Avaliação do lead } \\
\text { time total de } \\
\text { montagem }\end{array}$ & $\begin{array}{l}\text { o objetivo é medir a evolução do lead time total de } \\
\text { montagem de máquinas em comparação com o lead time } \\
\text { antes de implantar o sistema de produção enxuta }\end{array}$ & $\begin{array}{l}\text { lead time anterior de montagem } \\
\text { de máquinas e lead time atual de } \\
\text { montage m de máquinas }\end{array}$ \\
\hline $\begin{array}{l}\text { Movimentação na } \\
\text { montagem final }\end{array}$ & $\begin{array}{l}\text { visa avaliar a redução de movimentação de peças e } \\
\text { operários na montagem final com a implantação do projeto } \\
\text { enxuto }\end{array}$ & $\begin{array}{l}\text { movimentação atual e } \\
\text { movimentação antes do projeto }\end{array}$ \\
\hline Giro de estoque & $\begin{array}{l}\text { o objetivo é avaliar a eficiência da compra de inventários } \\
\text { em relação à sua utilização }\end{array}$ & $\begin{array}{l}\text { compra de materiais / quantidade } \\
\text { no estoque }\end{array}$ \\
\hline $\begin{array}{l}\text { Utilização de horas } \\
\text { disponíveis de mão- } \\
\text { de-obra }\end{array}$ & $\begin{array}{l}\text { o objetivo é comparar a quantidade mensal de horas } \\
\text { disponíveis de mão-de-obra com a quantidade de horas } \\
\text { necessárias para montar as máquinas }\end{array}$ & $\begin{array}{l}\text { horas disponíveis no mês / horas } \\
\text { necessárias para montagem de } \\
\text { máquina no mês }\end{array}$ \\
\hline $\begin{array}{l}\text { Avaliação do } \\
\text { número de } \\
\text { componentes } \\
\text { carregados por dia }\end{array}$ & $\begin{array}{l}\text { o objetivo é comparar o número de peças expedidas } \\
\text { diariamente com uma meta estipulada }\end{array}$ & $\begin{array}{l}\text { número de componentes } \\
\text { expedidos por dia }\end{array}$ \\
\hline $\begin{array}{l}\text { Número de } \\
\text { produtos montados } \\
\text { acima do tempo } \\
\text { pitch }\end{array}$ & $\begin{array}{l}\text { o objetivo é avaliar a eficiência das equipes de montagem } \\
\text { final em cumprir o tempo estabelecido pelo pitch (significa } \\
\text { o tempo que é necessário para completar um contêiner de } \\
\text { produtos em uma área da produção) }\end{array}$ & $\begin{array}{l}\text { número de produtos montados } \\
\text { dentro do tempo pitch }\end{array}$ \\
\hline $\begin{array}{l}\text { Eficiência do setor } \\
\text { "gargalo" }\end{array}$ & $\begin{array}{l}\text { o objetivo é medir a eficiência diária do setor que restringe } \\
\text { o ritmo de produção diariamente }\end{array}$ & $\begin{array}{c}\text { produtos acabados / programação } \\
\text { da produção diária }\end{array}$ \\
\hline $\begin{array}{l}\text { Atendimento de } \\
\text { cartões }\end{array}$ & $\begin{array}{l}\text { o objetivo é comparar o número de cartões comprados, } \\
\text { número de cartões atendidos e o número de cartões } \\
\text { vermelhos }\end{array}$ & $\begin{array}{l}\text { contagem de cada um dos tipos de } \\
\text { cartões }\end{array}$ \\
\hline $\begin{array}{l}\text { Número de itens no } \\
\text { vermelho x milk run }\end{array}$ & $\begin{array}{l}\text { o objetivo é medir a eficiência do novo sistema milk run } \\
\text { implantado. }\end{array}$ & $\begin{array}{l}\text { número de cartões no vermelho do } \\
\text { milk run e o número de cartões } \\
\text { total no vermelho }\end{array}$ \\
\hline
\end{tabular}


Essa lista de indicadores de desempenho foi reunida metodicamente a partir de entrevistas com os participantes dos projetos de produção enxuta (membros do grupo de pesquisa de Gestão e Mudança Organizacional (GMO), do Núcleo de Manufatura (ver www.numa.org.br) da Escola de Engenharia de São Carlos) e publicações sobre esses projetos (PASSAGEM, 2003).

Analisando-se tais pesquisas, ficou claro que os indicadores utilizados pelo Sistema de Produção Enxuto podem ser classificados em dois tipos: estratégicos ou de projeto (informam como as novas práticas são utilizadas pelas pessoas e quais são os efeitos do projeto enxuto nos indicadores financeiros) e os operacionais ou de processo (são indicadores de controle para mensurar a eficiência e eficácia dos processos individuais ).

Todas as empresas que implantaram o projeto enxuto desenvolveram e passaram a utilizar novos indicadores de desempenho. Ao mesmo tempo, criaram meios (Gestão Visual) para divulgar a informação aos usuários e outras áreas operacionais e administrativas.

\section{COMENTÁRIOS FINAIS}

No processo de elaboração e implantação dos indicadores de desempenho prevaleceu a experiência das pessoas que participaram do projeto de produção enxuta. Nota-se que em nenhum dos casos relatados nas entrevistas e publicações foi utilizado um método ou procedimento sistemático para desenvolver o sistema de avaliação de desempenho para o novo sistema de manufatura.

É importante ressaltar que isso não significa que o sistema deixou de apresentar resultados satisfatórios para as empresas, porém, esse tipo de atitude pode levar a criar um número elevado de indicadores, levantar informações desnecessárias do processo produtivo, concluir precipitadamente ou até mesmo errar sobre o desempenho dos processos, falhar na integração dos indicadores estratégicos e operacionais, entre outros aspectos, dificultando a avaliação ou gestão do desempenho. 
O quadro 3 indica as diferenças que existem no processo de medição de desempenho do sistema de produção em massa e o sistema de produção enxuto. As mudanças surgem pelas próprias características dos processos de manufatura. No sistema de produção em massa $a_{2}$ produção é baseada na previsão da demanda (gerando estoques elevados de produtos acabados ou intermediários), e no sistema de produção enxuto a produção é puxada, ou seja, só é produzido um determinado produto quando existe uma demanda real.

Quadro 3: Diferenças do processo de medição de desempenho nos sistemas de manufaturas

\begin{tabular}{|c|c|}
\hline Sistema de Produção em Massa & Sistema de Produção Enxuto \\
\hline $\begin{array}{l}\text { - O sistema de indicadores de desempenho é utilizado } \\
\text { para medir a eficiência dos processos, a produtividade da } \\
\text { linha, o absenteísmo dos funcionários, a quantidade de } \\
\text { refugo, número de acidentes etc., informações que às } \\
\text { vezes não demonstram a situação real da empresa diante } \\
\text { dos seus acionistas, fornecedores e clientes. } \\
\text { - O sistema valoriza apenas os índices específicos } \\
\text { locais, desconsiderando a visão global de todo o processo } \\
\text { produtivo. } \\
\text { - Nesse sistema de produção é comum utilizar meios } \\
\text { informais para medir o desempenho dos processos } \\
\text { produtivos. } \\
\text { - Na maioria das vezes os dados divulgados nos setores } \\
\text { da empresas são desatualizados e falham na integração. } \\
\text { - As informações são centralizadas em aspectos que não } \\
\text { necessariamente monitoram e avaliam a satisfação do } \\
\text { cliente final. }\end{array}$ & $\begin{array}{l}\text { - Os indicadores de desempenho criados a partir das } \\
\text { atividades que agregam valor permitem monitorar } \\
\text { constantemente os resultados individuais de cada um dos } \\
\text { processos e globais do fluxo de valor. } \\
\text { - A análise visual (gestão visual) do fluxo de valor do } \\
\text { produto é valorizada pelos gerentes e colaboradores } \\
\text { (funcionários) da empresa. Neste caso, à visão de todas as } \\
\text { atividades permite remover as restrições e melhorar o } \\
\text { fluxo de valor. } \\
\text { - O sistema de indicadores de desempenho passa a } \\
\text { determinar as fontes de variação e atividades que não } \\
\text { agregam valor aos clientes. Consequentemente, os } \\
\text { desperdícios podem ser identificados e eliminados. } \\
\text { - Os indicadores de desempenho permitem analisar e } \\
\text { avaliar a situação atual da empresa. Com isso é possível } \\
\text { diagnosticar e direcionar as ferramentas de melhoria } \\
\text { contínua para as áreas que apresentam resultados } \\
\text { insatisfatórios. } \\
\text { - Os resultados dos eventos de melhoria realizados no } \\
\text { fluxo de valor são medidos. Formalizando o sistema de } \\
\text { informação interno da empresa. }\end{array}$ \\
\hline
\end{tabular}

A produção enxuta é um novo paradigma que vêm se formando e crescendo nas empresas manufatureiras e de serviços. Entretanto, o tema é incipiente no país e carece de pesquisas. Entre os projetos de pesquisa se destacam os realizados pela Universidade de Campinas, Universidade de São Paulo (GMO/NUMA), Universidade Federal de Santa Catarina, Universidade Federal de São Carlos e do Lean Institute Brasil. Acredita-se que várias mudanças ainda ocorreram nos conceitos, principalmente, quando se levam em consideração as peculiaridades (barreiras culturais, falta de investimentos, resistência à mudança etc.) do meio empresarial brasileiro. 


\section{REFERÊNCIAS}

Bititci, U.S., Carrie, A.S.; McDevitt, L. Integrated performance measurement systems: a development guide, Int. J. Op. \& Prod. Management, v.17, n.5, p.522-534, 1997.

Bititci, U.S., Suwignjo, P.; Carrie, A.S. Strategy management through quantitative modeling of performance measurement systems, Int. J. Prod. Economics, v.69, p.15-22, 2001.

Cardoza, E., Carpinetti, L.C.R. e Martins, R.A. Estudo sobre o processo de implementação de sistemas de medição de desempenho em empresas manufatureiras, XXIII Encontro Nacional de Engenharia de Produção, Ouro Preto, MG, 2003.

Cooney, R., Is "lean" a universal production system? Batch production in the automotive industry, Int. J. Op. \& Prod. Management, v.22, n.10, p.1130-1147, 2002.

Cross, K.F.; Lynch, R.L. Managing the corporate warriors, Quality progress, v.23, n.4, p.54-59, 1990.

Cusumano, M.A., The Japanese automobile industry, Cambridge: The Council on East Asian Studies, Harvard University, p.262-319, 1989.

Flapper, S.D.P.; Fortuin, L.; Stoop, P.P.M. Towards consistent performance management systems, Int. J. Op. \& Prod. Management, v.16, n.7, p.27-37, 1996.

Kaplan, R.S.; Norton, D.P. Using the balanced scorecard as a strategic management system, Harvard business review, v.74, n.1, p.75-85, Jan./Feb, 1996.

Lewis, M.A. Lean production and sustainable competitive advantage, Int. J. of Op. \& Prod. Management, v.20, n.8, p.959-978, 2000.

Martins, R.A. The use of performance measurement information as a drive in designing a performance measurement system, Proceedings of the third performance measurement and management conference, Boston, MA, p.371-378, 2002.

Neely, A. Measuring business performance, The Economics, London, c.3, p.70-89, 1998.

Neely, A., Mills, J., Platss, K., Richards, H., Gregory, M., Bourne, M.; Kennerley, M., Performance measurement system design: developing and testing a process-based approach, Int. J. of Op. \& Prod. Management, v.20, n.10, p.1119-1145, 2000.

Neely, A.; Adams, C. Perspectives on performance: The performance Prism, Center for business performance, Cranfield, UK, 2001.

Ohno, T. O sistema Toyota de produção além da produção em larga escala, Trad. Cristina Schumacher, Artes Médicas, Porto Alegre, 145p., 1997.

Passagem, G.Z. Estudo e implementação de um sistema de produção enxuta em uma empresa do ramo agroindustrial, Monografia (Graduação), EESC-USP, 2003.

Rentes, A.F., Carpinetti, L.C.R.; Van Aken, E.M. Measurement system development process: a pilot application and recommendations, Proceedings of the third performance measurement and management conference, Boston, MA, p.487-494, 2002.

Rother, M.; Shook, J. Aprendendo a enxergar: mapeando o fluxo de valor para agregar valor e eliminar o desperdício, The Lean Institute Brasil, São Paulo,1999.

Sanchez, A.M.; Perez, M.P. Lean indicators and manufacturing strategies, Int. J. of Op. \& Prod. Management, v.21, n.11, p.1433-1451, 2001.

Standard, C.; Davis, D. Running today's factory: a proven strategy for lean manufacturing, Hanser Gardner Publications, Cincinnati, 1999.

Van Aken, E.M., Klein, S.; Groesbeck, R. Building better performance measurement systems, Enterprise engineering research Lab., Blacksburg, VA, Virginia Tech, 2001.

Warnecke, H.J.; Hüser, M. Lean production, Int. J. Production Economics, v.41, p.37-43, 1995.

Womack, J.P., Jones, D.T.; Roos, D. A máquina que mudou o mundo, Campus, Rio de Janeiro, 1992. 
Artigo Recebido em 17/05/04 e aceito para publicação em 01/07/05. 